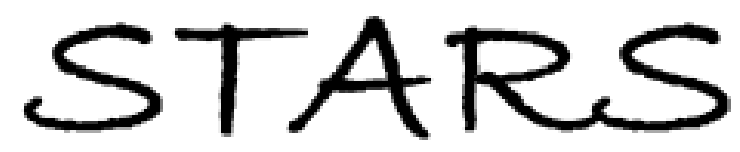

University of Central Florida

STARS

$1-1-1997$

\title{
Transfer of momentum and torque from a light beam to a liquid
}

\author{
A. Yu. Savchenko \\ University of Central Florida \\ N. V. Tabiryan \\ University of Central Florida \\ B. Ya. Zel'dovich \\ University of Central Florida
}

Find similar works at: https://stars.library.ucf.edu/facultybib1990

University of Central Florida Libraries http://library.ucf.edu

This Article is brought to you for free and open access by the Faculty Bibliography at STARS. It has been accepted for inclusion in Faculty Bibliography 1990s by an authorized administrator of STARS. For more information, please contact STARS@ucf.edu.

\section{Recommended Citation}

Savchenko, A. Yu.; Tabiryan, N. V.; and Zel'dovich, B. Ya., "Transfer of momentum and torque from a light beam to a liquid" (1997). Faculty Bibliography 1990s. 2087.

https://stars.library.ucf.edu/facultybib1990/2087

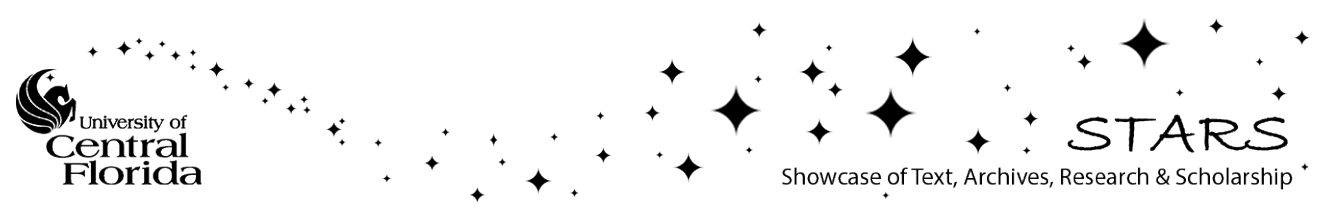




\title{
Transfer of momentum and torque from a light beam to a liquid
}

\author{
A. Yu. Savchenko, N. V. Tabiryan, and B. Ya. Zel'dovich* \\ CREOL and Physics Department, University of Central Florida, Orlando, Florida 32816-2700 \\ (Received 26 February 1997; revised manuscript received 5 May 1997)
}

\begin{abstract}
Refraction or absorption of light results in the force and torque, i.e., transfer of momentum and angular momentum from light to the medium. In transversely inhomogeneous beams, the force per unit volume $\mathbf{f}$ may have curlf $\neq \mathbf{0}$ leading to flow or to nonthermal and nongravitational convection in liquids. The force and the torque in scattering systems are as strong as in absorbing materials and may allow one to carry out experiments avoiding thermal effects. Nonlinear optical response of liquid crystals due to this convection is discussed. [S1063-651X(97)03210-8]
\end{abstract}

PACS number(s): 42.70.Df, 42.50.Vk, 42.65.Sf, 47.27.-i

\section{INTRODUCTION}

The pressure of light and the problem of the proper choice of the expression for the electromagnetic-field momentum density and stress tensor in a medium was a subject of hot discussions since the works of Lorentz, Abraham, and Minkowski; see the relatively modern studies [1,2]. The existence of light pressure is especially evident if the light is considered as a beam of quanta with the flux density

$$
\text { I }\left(\text { quanta } / \mathrm{m}^{2} \mathrm{~s}\right)=\left[\mathbf{S}\left(\mathrm{W} / \mathrm{m}^{2}\right)\right] /[\hbar \omega(\mathrm{J})] .
$$

Here and below $\mathbf{S}=\varepsilon_{0} c^{2} \mathbf{E} \times \mathbf{B}$ is the time-averaged value of the Poynting vector, $\hbar \omega$ and $\hbar \mathbf{k}=(\hbar \omega n / c) \mathbf{k} / k$ are, respectively, the energy and the momentum of a photon, $n$ is the refractive index, and $k=\omega / c$ in vacuum. (In [2], this is referred to as Minkovski's form of "crystal momentum", or " "pseudomomentum.') Then the reflection of a beam by a surface back into vacuum results in the buildup of the pressure $\Pi$ (force $F$ per area $A$ ),

$$
\Pi=F / A=2 \hbar k I=2 S k / \omega=2 S / c .
$$

This fact, evident nowadays, was verified experimentally in 1900 by Lebedev [3]; see also [4]. A lot of work on the transfer of momentum and torque from the light to individual atoms, molecules, and macroscopic particles has been performed so far; see, e.g., [5-7]. The main concern of those works was the "differential" motion of the individual particles with respect to the surrounding medium.

Transfer of the "spin" part of the angular momentum of light to the director of a nematic liquid crystal (NLC) was observed by Santamato et al. [8]. In that work the rotation of the NLC director was observed, with the angular velocity $\Omega / 2 \pi$ about $0.02 \mathrm{~Hz}$; the power density of the argon laser beam was about $2 \times 10^{3} \mathrm{~W} / \mathrm{cm}^{2}$ and the thickness of the cell was $65 \mu \mathrm{m}$.

Transfer of the "orbital" angular momentum from a laser beam with a wave-front dislocation (with a phase singularity) to an absorptive particle was observed directly in the

*FAX: (407) 823-6880.

Electronic address: boris@mail.creol.ucf.edu experiment, which was reported in [9]. The LaguerreGaussian beam proportional to $\exp (-i \omega t+i k z+i m \varphi)$ with $m= \pm 3$ was used and the particles of the size about $2.5 \mu \mathrm{m}$ acquired the rotation speed $\Omega / 2 \pi$ about $4 \mathrm{~Hz}$. Smaller particles were reported to join the rotation of the main trapped particle. The above value of the rotation speed was in agreement with the idea of the balance between the two contributions to the torque. The first one is equal to $m P / \omega$ $=\hbar m P / \hbar \omega$, where $P$ is the power absorbed by the particle, and the second contribution $-6 \pi \eta \Omega r^{3}$ is due to viscous friction (viscosity coefficient $\eta$ ) of the liquid surrounding an almost spherical particle of the radius $r$.

An important development of those measurements was reported in [10], where the $z$ component of the orbital angular momentum $m=3$ of the absorbed photon was either increased $m+\sigma=3+1=4$ or decreased $m+\sigma=3-1=2$ by the spin angular momentum of the photon $\sigma=S_{z} / \hbar= \pm 1$. That was achieved by switching the circular polarization from right to left. An angular velocity $\Omega / 2 \pi$ about $1 \mathrm{~Hz}$ was observed and the theoretically predicted ratios 4:3:2 were confirmed in the experiment.

Many theoretical results were obtained in the papers by Allen and co-workers [11-13], concerning the relative contributions of orbital and spin angular momenta. Reference [12] contains the discussion of the nonparaxial approximation in the theory of the angular momentum of a light beam.

In the present paper we discuss the force and study the torque transferred from light to the volume of the medium as a whole. At first glance it may seem that the corresponding effects are almost undetectable due to the presence of a large quantity $c=3 \times 10^{8} \mathrm{~m} / \mathrm{s}$ in the denominator of the expression for the pressure $2 S / c$, Eq. (2). However, just those effects are responsible for the giant orientational optical nonlinearity of liquid crystals (LCs); see, e.g., [14-20]. We show here that the action of light pressure on a liquid as a whole is both remarkable and easily detectable. The emphasis of the present work is not on the deformation of the profile of the free surface of liquid under the light pressure (see [2-4] and references therein), but on the creation of steady-state convective flow of the liquid in a confined volume. It may also be called "convective motion." We would like to emphasize here that this is not "thermal convection in the presence of gravity," i.e., this is not the "convection", in the most usual sense of this word. The word "convection" in our case is 


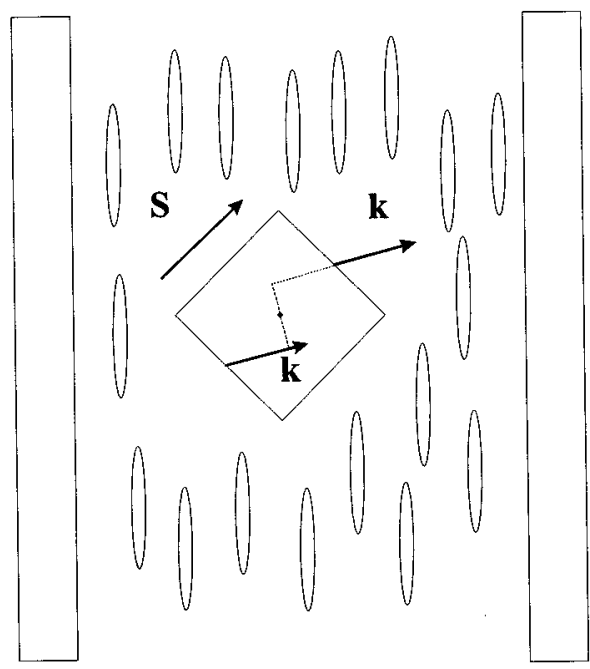

FIG. 1. Orientation of the liquid crystal as arising from the noncollinearity of the Poynting vector $\mathbf{S}$ and photon momentum vector $\hbar \mathbf{k}$.

applied to remind the reader that we discuss essentially circulatory flow of a liquid as opposed, for example, to the monodirectional Poiseuille-type flow.

In other words, we have not rediscovered that the light pressure is stronger than it was assumed to be. It is the "softness" of liquids making them highly responsive to even minute forces that seems so fascinating to us. Besides, we want to emphasize the importance of the acting force to have nonzero curl. Namely, the effect of the well-known (and large) gravity force on a homogeneous liquid cannot result in flow or in convection for the simple reason that the potential character of this force leads to the buildup of the appropriate hydrostatic pressure, which completely balances the gravity in the steady state.

The source of inspiration for the present work was the observation of the optical processes in dye-doped (i.e., absorbing) NLCs [21-23], where the nonlinearity turned to be nearly 100 times larger than for the "standard giant" nonlinearity [14-20]. Originally, we attempted to relate that enhancement to the light-induced convection mechanism. We think now that convection was not responsible for the phenomena observed in [21-23]. Nevertheless, the results of the present work show that the steady-state flow of a liquid, induced by transfer of momentum and torque from light to a liquid (and liquid crystals, in particular), definitely exists as an independent and strong phenomenon.

\section{LIQUID-CRYSTAL ORIENTATIONAL NONLINEARITY AS A LIGHT PRESSURE EFFECT}

Consider a light beam propagating in a transparent NLC, i.e., in an optically uniaxial crystal. An extraordinary wave propagates here in such a way that the group-velocity vector (or the Poynting vector $\mathbf{S}$ ) is at an angle $\beta$ from the direction of the wave vector $\mathbf{k}$. Taking a small cube of the size $a \times a$ $\times a$ with the direction $z^{\prime}$ along the Poynting vector $\mathbf{S}$ (see Fig. 1), one is ensured that the photons are entering the cube through the face $z^{\prime}=-a / 2$ only and are quitting the cube through the face $z^{\prime}=+a / 2$ only. Since the medium is trans-

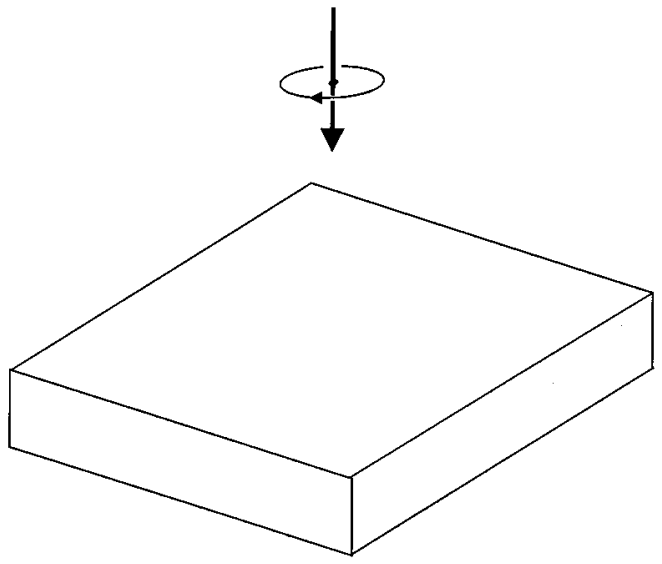

FIG. 2. Rotation of a liquid due to the transfer of the photon's spin angular momentum via absorption of circularly polarized light.

parent, there is a complete balance between the input and output numbers of quanta, energy, and momentum. However, the deviation of the $\mathbf{k}$ direction from the $\mathbf{S}$ direction gives rise to the "lever arms," $+\beta a / 2$ for the input quanta and $-\beta a / 2$ for the output quanta, and the torque $T$ applied to the volume $V=a^{3}$ equals

$$
T=a^{2} I \hbar k(a \beta)=V \beta S n / c .
$$

On the other hand, the standard expression for the torque is

$$
\mathbf{T}=\mathbf{r} \times \mathbf{F}=\mathbf{r} \times e \mathbf{E}=e \mathbf{r} \times \mathbf{E}=\mathbf{d} \times \mathbf{E}=V \mathbf{P} \times \mathbf{E}=V \mathbf{D} \times \mathbf{E},
$$

where $\mathbf{P}=\mathbf{D}-\varepsilon_{0} \mathbf{E}$ is the dipole moment of unit volume. Since $\mathbf{D}=\varepsilon_{0} c^{2} \mathbf{k} \times \mathbf{B} / \omega$ and $\mathbf{E}=\mathbf{S} \times \mathbf{B} / B^{2} \varepsilon_{0} c^{2}$ in a plane wave, the angle between $\mathbf{D}$ and $\mathbf{E}$ has the same value $\beta$ as the angle between $\mathbf{S}$ and $\mathbf{k}$ and the "quantum" and standard expressions for the torque evidently coincide.

\section{ROTATION OF A LIQUID DUE TO ABSORPTION OF THE PHOTON'S SPIN}

If the medium has nonzero absorption coefficient $\alpha\left(\mathrm{m}^{-1}\right.$, for intensity), then the momentum $\hbar \mathbf{k}$, orbital angular momentum $\mathbf{r} \times \hbar \mathbf{k}$, and spin angular momentum $\hbar \sigma \mathbf{k} / k$ of the photons may be deposited into the small volume $V$ of the medium: the force $\mathbf{F}=\mathbf{f} V=\alpha V S \mathbf{k} / \omega(\mathrm{N})$ and the spin torque $\mathbf{T}=\tau V=V \alpha S \sigma \mathbf{k} / k \omega(\mathrm{N} \mathrm{m})$. Here $\sigma= \pm 1$ for the right or left circular polarization, respectively. We do not consider orbital torque in this section.

Let a circularly polarized light of a constant intensity $S_{z}(x, y, z)=S=$ const illuminate a cell with weakly absorbing liquid $\alpha L \ll 1$ occupying the space $0 \leqslant z \leqslant L$ (Fig. 2). The transverse boundary of the axially symmetric beam $x^{2}+y^{2}$ $=R_{0}^{2}$ is assumed to be very distant from the region of interest. Taking $\rho$ as the density of liquid and the azimuthal velocity $u_{\varphi}(r, t)$ as the measure of the angular momentum $\Delta M$ of the hydrodynamic motion in the small layer of liquid 0 $\leqslant z \leqslant L, \quad 0 \leqslant \varphi \leqslant 2 \pi, r \leqslant r^{\prime} \leqslant r+\Delta r$, one may write $\Delta M$ $=2 \pi z_{0} r^{2} \rho u_{\varphi} \Delta r$. Then the equation for $d \Delta M / d t$ expresses the balance between the angular momentum acquired from light and the viscous transfer of angular momentum $M$ from 
one layer to the other. Neglecting the friction with the walls $z=0$ and $L$, one gets

$$
\frac{\partial u_{\varphi}}{\partial t}-\frac{D}{r^{2}} \frac{\partial}{\partial r}\left[r^{3} \frac{\partial}{\partial r}\left(\frac{u_{\varphi}}{r}\right)\right]=\frac{B}{r}, \quad B=\frac{\sigma \alpha S}{\rho \omega}, \quad D=\frac{\eta}{\rho} .
$$

Here $\eta\left(\mathrm{N} \mathrm{s} / \mathrm{m}^{2}\right)$ is the viscosity coefficient and $D$ $=\eta / \rho\left(\mathrm{m}^{2} / \mathrm{s}\right)$ is the kinematic viscosity (of the dimensions of a diffusion coefficient).

It is interesting to note that this partial differential equation with zero initial condition $u_{\varphi}(r, t=0)=0$ and with instantaneous switch-on of the spatially homogeneous and time-independent illumination, $S=0$ at $t=0$ and $S=$ const at $t>0$, has a self-similar solution. Introducing the dimensionless independent variable (argument) $\xi=r /(4 D t)^{1 / 2}$ and the new dependent variable (function) $w(\xi)=u_{\varphi} / r$, one gets the equation

$$
\xi^{2} \frac{d^{2} w}{d \xi^{2}}+\left(3 \xi+2 \xi^{3}\right) \frac{d w}{d \xi}=-C, \quad C=\frac{\sigma \alpha S}{\omega \eta} .
$$

Here $\xi>0$ and the boundary conditions are $w(\xi \gg 1)$ $\approx C / 4 \xi^{2}$ and $w(\xi \ll 1) \approx-(C / 2) \ln \xi$. Indeed, one can neglect the viscosity at large radius, i.e., at $\xi \gg 1$, and then the first boundary condition becomes evident directly from the Eq. (5). This limit is also equivalent to keeping only the term $2 \xi^{3} d w / d \xi$ on the left-hand side of Eq. (6). On the other hand, at small radius or at relatively large time, one can neglect the term $2 \xi^{3} d w / d \xi$ and the asymptotic behavior $w(\xi \ll 1) \approx-C \ln \xi$ becomes evident. This asymptotic limit corresponds to the almost "rigid-body" rotation $u_{\varphi}(r, t)$ $\approx \Omega(t) r$ with the slowly increasing angular velocity of rotation $\Omega(t) \approx(C / 2) \ln t$.

The explicit form of the self-similar solution is

$$
\omega(\xi)=\frac{C}{2} \int_{\xi}^{\infty}\left(1-e^{-t^{2}}\right) \frac{d t}{t^{3}}
$$

or, in slightly different notations,

$$
\begin{gathered}
w(\xi)=\frac{C}{4 \xi^{3}}\left[\operatorname{Ei}\left(1, \xi^{2}\right) \xi^{2}+1-e^{-\xi^{2}}\right], \\
\operatorname{Ei}\left(1, \xi^{2}\right)=\int_{1}^{\infty} e^{-i \xi^{2}} \frac{d t}{t}
\end{gathered}
$$

The graph of the dimensionless function $w(\xi) / C$ versus the dimensionless argument $\xi$ follows very nearly both of the asymptotic expressions: at $\xi \leqslant 1$ and at $\xi \geqslant 1$. For the given time $t$, the velocity $u_{\varphi}=r w(\xi)$ has its maximum $u_{\varphi}$ $\approx 0.5 C(D t)^{1 / 2}$ in a rather broad interval $0.5<\xi<1$ of values of $\xi$.

An approximate way to take into account the decelerating influence of the boundaries $z=0$ and $L$ is to add the term $D d^{2} u_{\varphi} / d z^{2}$ to the right-hand side of Eq. (5). Assume also the approximate $z$ profile of the velocity as $u_{\varphi} \sin (\pi z / L)$. Then one may describe the friction effect of the wall as the term $-\Gamma u_{\varphi}$ added to the right-hand side of Eq. (5), where the inverse relaxation time $\Gamma=1 / \tau$ is

$$
\Gamma=1 / \tau=D(\pi / L)^{2}
$$

For the radius $r \geqslant L / \pi$, one should consider mostly the friction with the walls and not between the layers with the different $r$. Then the steady-state solution for the velocity is

$$
u_{\varphi}=B / r \Gamma=L S(\alpha L) / \pi^{2} \omega \eta r .
$$

The original assumption of small absorption means that the factor $\alpha L$ cannot be made larger than 1 in Eq. (8). Let us make some numerical estimations, taking $\alpha L=1$ and $r=L$ in Eq. (8) and $\eta \approx 10^{-2} \mathrm{P}=10^{-3} \mathrm{~N} \mathrm{~s} / \mathrm{m}^{2}$ (for water), and $\omega=3.7 \times 10^{15} \mathrm{rad} / \mathrm{s}$ for $\lambda=0.5 \mu \mathrm{m}$. Taking $S=10^{2} \mathrm{~W} / \mathrm{cm}^{2}$ $=10^{6} \mathrm{~W} / \mathrm{m}^{2}$, one gets $u_{\varphi}=5 \times 10^{-8} \mathrm{~m} / \mathrm{s}=0.025 \mu \mathrm{m} / \mathrm{s}$. This is evidently a very small effect. One cannot further increase the power density of radiation in the absorbing medium. Indeed, taking the thermal diffusivity coefficient about $\chi$ $\approx 1.5 \times 10^{-7} \mathrm{~m}^{2} / \mathrm{s}$ for water and the thickness $L$ of the cell about $100 \mu \mathrm{m}$, one gets the thermal relaxation time about $\tau$ $\approx L^{2} / \chi \pi^{2} \approx 0.7 \times 10^{-2} \mathrm{~s}$, so that the temperature increase at $\alpha S=S / L=10^{4} \mathrm{~W} / \mathrm{s} \mathrm{cm}^{3}$ is already about $15^{\circ} \mathrm{C}$.

However, one may use a medium with strongly scattering (but nonabsorbing) particles. In that case the medium still takes the spin of the incident radiation, but not its energy. Then a laser beam with the power $10 \mathrm{~W}$ focused into a spot $100 \times 100 \mu \mathrm{m}^{2}$ gives $S$ about $10^{5} \mathrm{~W} / \mathrm{cm}^{2}$ and the effect becomes three orders of magnitude stronger, i.e., $u_{\varphi}=5$ $\times 10^{-5} \mathrm{~m} / \mathrm{s}=2.5 \mu \mathrm{m} / \mathrm{s}$. Such a velocity may be easily observed by heterodyne registration of the Doppler shift of the scattered radiation.

We will see in the next section, however, that the velocity of the motion induced by the deposition of the photon's momentum itself, or of the $x$ and $y$ components of the "orbital" angular momentum, may give much larger effects. An estimation of the "gain" factor for the "orbital" torque in comparison with the "spin" torque is $2 \pi L / \lambda$, where $L$ is characteristic size either of the beam or of the cell. It corresponds to the notion that the lever arm for the spin of a photon is very small: about $\lambda / 2 \pi$.

\section{FLOW OF A LIQUID INDUCED BY LIGHT PRESSURE}

Consider now the linearized equations for the hydrodynamics of incompressible liquid under the influence of an externally applied force $\mathbf{f}(x, y, z)\left(\mathrm{N} / \mathrm{m}^{3}\right)$; see, e.g., [24-26]. In this section we neglect the spin part of the torque transferred from light to the unit volume of the liquid. Then one gets the Navier-Stokes-type equations

$$
\frac{\partial u_{i}}{\partial t}=D\left(\frac{\partial^{2}}{\partial x^{2}}+\frac{\partial^{2}}{\partial y^{2}}+\frac{\partial^{2}}{\partial z^{2}}\right) u_{i}+\frac{1}{\rho}\left(f_{i}-\frac{\partial}{\partial x_{i}} p(x, y, z, t)\right) .
$$

Here $p(\mathbf{r}, t)$ is the pressure and the velocity vector $\mathbf{u}$ satisfies the continuity equation, which, for an incompressible liquid, takes the form

$$
\frac{\partial u_{x}}{\partial x}+\frac{\partial u_{y}}{\partial y}+\frac{\partial u_{z}}{\partial z}=0 .
$$


The boundary conditions are zero for each of the three components of the velocity at the walls of the cell:

$$
\mathbf{u}(z=0, x, y, t)=\mathbf{u}(z=L, x, y, t)=\mathbf{0} .
$$

Homogeneity of the cell in the ( $x$ and $y)$ directions makes it natural to use a two-dimensional Fourier expansion for the solution of this problem:

$$
u(x, y, z, t)=\iint e^{i k_{x} x+i k_{y} y} U\left(k_{x}, k_{y}, z, t\right) d k_{x} d k_{y},
$$

where $u$ is any of $u_{x}, u_{y}, u_{z}, f_{x}, f_{y}, f_{z}, p$. It is natural to decompose the force and the velocity distributions into two parts: the parts $\mathbf{f}_{\perp}, \mathbf{u}_{\perp}$, which lie in the $(x, y)$ plane and are perpendicular to the two-dimensional $\mathbf{k}$ vector, and the rest of the $\mathbf{f}$ and $\mathbf{u}$ vectors.

The continuity equation is satisfied automatically for the components of velocity perpendicular to the wave vector $\mathbf{k}$ and then the natural expansion for those components is

$$
u(z, t), f(z, t)=\sum_{m=1}^{\infty}\left(u_{m}(t), f_{m}(t)\right) \sin \left(\frac{\pi m z}{L}\right),
$$

so that the boundary conditions are satisfied. The equation of motion contains no pressure term and becomes

$$
\frac{d u_{m}}{d t}+\gamma_{m} u_{m}=\frac{1}{\rho} f_{m}, \quad \gamma_{m}=D\left(k^{2}+m^{2} \pi^{2} / L^{2}\right)
$$

and the steady-state response is $u_{m}=f_{m} / \rho \gamma_{m}$.

The consideration of that $(x, y)$ component of the velocity $\mathbf{u}$ that is parallel to $\mathbf{k}$ vector, as well as taking account of its $z$ component [as excited by the force distribution $\mathbf{f}(\mathbf{r}, t)$ ], is somewhat more complicated since here the pressure term works. For $\mathbf{k} \neq \mathbf{0}$, one may express the "longitudinal" component of the velocity $u_{l}=(\mathbf{u} \cdot \mathbf{k}) / k$ via $u_{z}$ with the use of Eq. (10):

$$
u_{l}=i k^{-1} \partial u_{z} / \partial z .
$$

The equation (9) for the longitudinal component $u_{l}$ allows one to eliminate the pressure $p$ from the subsequent equations. Finally, introducing a new function $\psi$, one may get the differential equation

$$
\begin{aligned}
& \psi\left(k_{x}, k_{y}, z, t\right)=\left(1-\frac{1}{k^{2}} \frac{\partial^{2}}{\partial z^{2}}\right) u_{z}\left(k_{x}, k_{y}, z, t\right), \\
& \frac{\partial \psi}{\partial t}-D k^{2}\left(1-\frac{1}{k^{2}} \frac{\partial^{2}}{\partial z^{2}}\right) \psi=\frac{1}{\rho}\left(f_{z}+\frac{\partial}{\partial_{z}} \frac{i \mathbf{k} \cdot \mathbf{f}}{k^{2}}\right)
\end{aligned}
$$

or, if only the steady state is of interest, one gets an even simpler equation for $u_{z}$ :

$$
\begin{gathered}
\Delta_{3} \Delta_{3} u_{z}(x, y, z)=H(x, y, z), \\
\eta H=\frac{\partial}{\partial z}\left(\frac{\partial f_{x}}{\partial x}+\frac{\partial f_{y}}{\partial y}\right)-\left(\frac{\partial^{2}}{\partial x^{2}}+\frac{\partial^{2}}{\partial y^{2}}\right) f_{z},
\end{gathered}
$$

$$
\Delta_{3}=\left(\frac{\partial^{2}}{\partial x^{2}}+\frac{\partial^{2}}{\partial y^{2}}+\frac{\partial^{2}}{\partial z^{2}}\right) .
$$

Here $\Delta_{3}$ denotes the three-dimensional Laplace operator. The advantage of the use of $u_{z}$ consists in the simplicity of the boundary conditions: at both boundaries, both the function itself and its $z$ derivative must be equal to zero. The first statement is the condition of no slipping of a viscous liquid and the second one uses the above condition and the incompressibility equation (10).

The solution of Eq. (17) may be achieved in a Fourier representation (with respect to $k_{x}, k_{y}$ ). Namely, the solution of the equation

$$
\left(\frac{\partial^{2}}{\partial z^{2}}-k^{2}\right)^{2} U\left(z, k^{2}\right)=H\left(z, k^{2}\right)
$$

with the boundary conditions of zero values of $U$ and $\partial U / \partial z$ both at $z=0$ and $L$ is

$$
U(z, \mathbf{k})=\int_{0}^{L} d z G\left(z, z^{\prime}, k^{2}\right) H\left(z^{\prime}, \mathbf{k}\right),
$$

where the Green's function $G\left(z, z^{\prime}, k^{2}\right)=G\left(z^{\prime}, z, k^{2}\right)$ satisfies the equation

$$
\left(\frac{\partial^{2}}{\partial z^{2}}-k^{2}\right)^{2} G\left(z, z^{\prime}, k^{2}\right)=\delta\left(z-z^{\prime}\right)
$$

and the same boundary conditions. The general expression for $G\left(z, z^{\prime}, k^{2}\right)$ is

$$
\begin{aligned}
G\left(z, z^{\prime}, k^{2}\right)= & \theta\left(z^{\prime}-z\right)\{A[k z \cosh (k z)-\sinh (k z)] \\
& +B k z \sinh (k z)\}+\theta\left(z-z^{\prime}\right)(C\{k(L-z) \\
& \times \cosh [k(L-z)]-\sinh [k(L-z)]\} \\
& +D k(L-z) \sinh [k(L-z)])
\end{aligned}
$$

Indeed, it is easy to verify that Eq. (21) is a solution at any $z \neq z^{\prime}$ and boundary conditions are satisfied. Four requirements, the continuity of $G, \partial G / \partial z$, and $\partial^{2} G / \partial z^{2}$ at $z=z^{\prime}$ and the condition of unit step of $\partial^{3} G / \partial z^{3}$ at $z=z^{\prime}$, allow one to find all the four coefficients $A, B, C$, and $D$. Particular expressions are simple in principle, but extremely cumbersome. We have calculated them analytically with the use of symbolic computation of MATHEMATICA 3.0; MATHCAD 6.0 also gave the same (complicated) answer.

We have used the analytical solution Eq. (19) to find [by numerical computation of the Fourier integral (12)] the distribution of the components $u_{x}, u_{y}, u_{z}$ of the velocity vector u. Figure 3 shows the distribution of $u_{z}$ and $u_{r}$ under illumination of the cell by a Gaussian beam with the intensity profile

$$
\begin{gathered}
S(x, y, z)=\left(\pi a^{2}\right)^{-1} P_{0} \exp \left[-\alpha z-\left(x^{2}+y^{2}\right) / a^{2}\right], \\
f_{z}=\alpha S n / c .
\end{gathered}
$$

Here $a$ is the radius of the beam, which is equal to the half width by the criteria $\exp (-1)$ of the maximum, and $P_{0}$ is the total power of the beam. Particular values of the parameters 


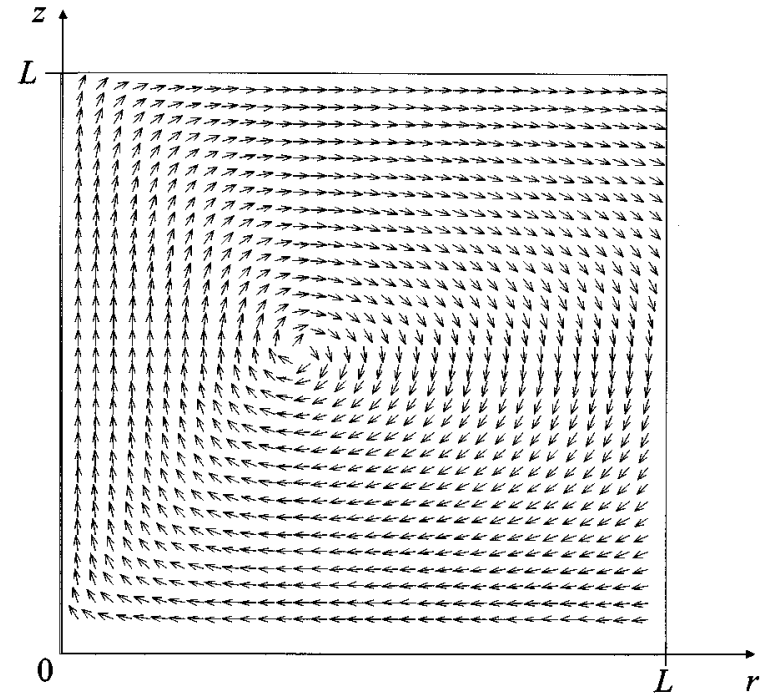

FIG. 3. Distribution of the velocity vector of the convective motion induced by the absorption (or scattering) of a Gaussian beam, normally incident in the $+z$ direction, versus radius $r$ from the beam axis and coordinate $z$ perpendicular to the cell's walls. The radius $a$ of the beam is the half width by the criteria $\exp (-1)$ of the maximum, $a=L / 4$, and the absorption $\alpha L=1$.

were $\alpha L=1$ and $a=0.25 L$ for Fig. 3. It should be noted that we have plotted all the small arrows with the same length in Fig. 3. Meanwhile, the values of velocity are the highest at the axis of the beam $r=0$. Actually, for $\alpha L=1$ the maximum of $u(r=0, z)$ is localized quite close to the middle of the cell $z=L / 2$.

The photons comprising the beam (22) have a zero $z$ component of the orbital angular momentum. Therefore, no rotation of the liquid around the $z$ axis is excited via the scattering or absorption of such a beam. However, the force $\mathbf{f}$ applied to a unit volume has a nonzero $z$ component. Therefore, the $x$ and $y$ components of the orbital torque, $\tau_{x}=y f_{z}$ and $\tau_{y}=-x f_{z}$, are transferred to the unit volume of the liquid. Sure enough, these components depend explicitly on the choice of the origin for the $x$ and $y$ coordinates. Moreover, the total torque is zero if that origin is chosen at the axis of the beam. It should be reminded, however, that we are interested in the motion of one part of the liquid relative to the other. Therefore, the axially localized $z$ component of the force yields a nonzero torque $\tau_{y}=-x f_{z}$ if the origin is chosen somewhere in the middle of Fig. 3. It is in this choice-of-origin noninvariant sense that we attribute the depicted circulatory flow of liquid to the transfer of the $x$ and $y$ components of the orbital torque from light to the liquid.

The numerical value of the maximum of the generated velocity $u_{z}$ for viscosity $\eta=10^{-2} \mathrm{P}$ (water) and $L$ $=100 \mu \mathrm{m}$ was $9 \mu \mathrm{m} / \mathrm{s}(0.0009 \mathrm{~cm} / \mathrm{s})$ for the beam power 2 $\mathrm{mW}$. Figure 4 shows the dependence of the value of the maximum of the generated velocity $u_{z}$ versus the dimensionless parameter $a / L$. We see a rather fast decrease of $u_{z}$ at larger radii of the beam. The explanation is that at $a / L \gg 1$ the force may be considered as almost potential.

Equation (17) is convenient for the estimation of the convection strength. Consider, for example, the case where the

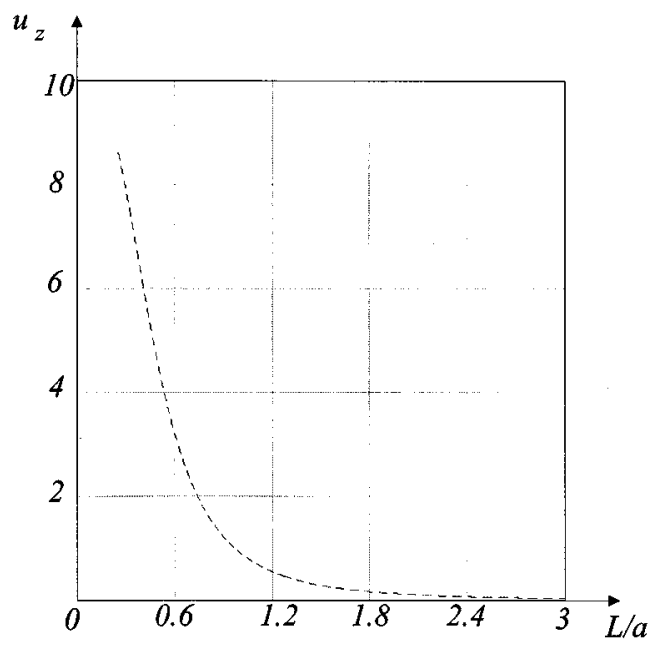

FIG. 4. Dependence of the maximum value of the $z$ component of the velocity of convective motion $(\mu \mathrm{m} / \mathrm{s})$ on the ratio $a / L$ for absorption $\alpha L=1$ and laser power $2 \mathrm{~mW}$.

transverse size $2 a$ of the exciting beam is about the thickness $L$ of the cell. Then the estimation for the spatial derivatives $\partial / \partial z$ is about $\pi / L$ and $\partial / \partial x, \partial / \partial y$ are about $1 / 2 a=1 / L$. As a result, one gets the estimation for the velocity of convection

$$
u \approx L S(\alpha L) n / \pi^{4} c \eta .
$$

We see that this value of the excited velocity is larger than the velocity for the "photon-spin"-induced rotation by the factor $2 \pi r / \lambda$, or about $2 \pi L / \lambda$. For the values $\alpha L=1, S$ $=100 \mathrm{~W} / \mathrm{cm}^{2}, L$ about $100 \mu \mathrm{m}$, and $\eta \approx 10^{-2} \mathrm{P}$ for water, one gets the velocity of about $5 \mu \mathrm{m} / \mathrm{s}$, i.e., in surprising agreement with the exact numerical calculations. Indeed, the accuracy of such an estimation is somewhat worse for large transverse sizes of the beam.

The Doppler shift of the $\lambda=0.5 \mu \mathrm{m}$ scattered radiation for the velocity of the particles $10 \mu \mathrm{m} / \mathrm{s}$ is about $u n / \lambda$ $=27 \mathrm{~Hz}$, an easily measurable quantity. One may further increase the value of the Poynting vector $S$ by three orders of magnitude in the experiment if the medium does not absorb light but merely scatters it. Then the effect becomes an extra three orders of magnitude stronger.

\section{LIGHT-INDUCED CONVECTION IN LIQUID CRYSTALS}

The motion of a liquid crystal is governed by the viscous stress tensor, which is much more complicated than NavierStokes expression for isotropic liquids. Therefore, we will not present those equations here and will discuss the corresponding effects only at the level of estimations. Viscosity coefficients both for reorientation and for hydrodynamic motion for a typical LC are about $1 \mathrm{P}$. The absorption coefficient may be made as large as necessary by the addition of dyes. However, for the elimination of heating, one may use the scattering of light. The first evident possibility is the admixture of the scattering (but nonabsorbing) particles. We want to note here specially that liquid crystals possess very strong intrinsic scattering of light by the thermodynamically equilibrium fluctuations of the director. For example, the ex- 
tinction coefficient due to scattering may be as large as $10 \mathrm{~cm}^{-1}$ for NLCs; see, e.g., [20]. Moreover, it was predicted in [21] and demonstrated experimentally [28] that the ordinary wave in a nematic LC may propagate without distortions and deflections at a rather high distance: up to 3 $\mathrm{cm}$ in an experiment similar to that in [28]. Then the only source of attenuation of the ordinary wave is molecular scattering, which leads to the transfer of momentum and torque from a light beam to the LC.

All the above estimations for the convection velocity, with the modification of the viscosity coefficient, are applicable to LCs. Hence, in the conditions $\alpha L=0.1, P$ $=2 \mathrm{~mW}, L$ about $100 \mu \mathrm{m}, \eta \approx 1 \mathrm{P}$ for LCs, and $a=L / 4$, one gets a velocity $u$ about $0.1 \mu \mathrm{m} / \mathrm{s}$ and the gradients of the velocity $\pi u / L$ about $0.003 \mathrm{~s}^{-1}$. This should be compared with the relaxation constant $\gamma$ about $1 \mathrm{~s}^{-1}$ for a $100-\mu \mathrm{m} \mathrm{LC}$ cell. Therefore, in the typical conditions of strong coupling between the gradients of the velocity and the orientation [14], the dimensionless degree of convection-induced reorientation may be about $\delta \theta \approx \pi u / L \gamma=0.003 \mathrm{rad}$ of the director deflection. The phase modulation of the light beam due to such a reorientation may be about $\delta \phi \approx 2 \pi \Delta n L \delta \theta / \lambda$, i.e., about $0.6 \mathrm{rad}$ or more, and hence is easily observable. Moreover, the above estimations correspond to the $2-\mathrm{mW}$ beam from a $\mathrm{He}-\mathrm{Ne}$ laser focused into a focal waist $50 \times 50$ $=\left(F W e^{-1} M\right)^{2} \mu \mathrm{m}^{2}$. Since our mechanism of convection exploits only "mechanical" (and not coherent) properties of light, one may use 5-W laser diode to increase the effect by an extra factor about 2500 .

\section{COMPARISON WITH THE MOTION OF INDIVIDUAL PARTICLES}

As was mentioned in the Introduction, our main interest is in the macroscopic motion of the liquid. Meanwhile, the subject of a large body of interesting publications in the field of light-induced motion (e.g., [5-13]) is the translational or rotational motion of an individual particle relative to the surrounding liquid. Here we will make the comparison of the corresponding values of the velocities.

The velocity $u_{\text {ind }}$ of an individual particle may be considered to be the result of the balance between the following two forces. The first one $f=\sigma S n / c$ is due to the scattering or absorption of light ( $\sigma$ is the corresponding cross section). The second force $f=-6 \pi \eta r u_{\text {ind }}$ is due to the viscous friction of an almost spherical particle of the radius $r$. A comparison of $u_{\text {ind }}=\sigma n S / 6 \pi \eta c r$ with the expression Eq. (23) for the macroscopic velocity of liquid yields

$$
\frac{u_{\text {macro }}}{u_{\text {ind }}} \approx 0.2 \frac{L^{2} \alpha}{r}=0.2 \frac{L^{2} N \sigma}{r} .
$$

We see that the velocity $u_{\text {ind }}$ of an individual scatterer or absorber (particle) is larger than $u_{\text {macro }}$ in the limit of a low density of particles and thin specimen (low $N$ and $L$ ), i.e., for an almost transparent liquid specimen, with very rare particles in it. On the contrary, we are interested in this paper in the case when $u_{\text {ind }} \ll u_{\text {macro }}$. Such a situation is realized for a relatively thick sample with a large number of scatterers. An example may be taken of the particles with the size $r$ $\approx 0.5 \mu \mathrm{m}, L=100 \mu \mathrm{m}, \sigma \approx \pi r^{2} \approx 1 \mu \mathrm{m}^{2}$, and $N L \sigma \approx 1$, so that $N \approx 10^{-2} \mu \mathrm{m}^{-3}$. (Note that it is only for the particle size about a wavelength that one may estimate $\sigma \approx \pi r^{2}$.) In that case $u_{\text {macro }} / u_{\text {ind }} \approx 40$ and the macroscopic circulatory flow (convection) of a liquid is much stronger than the motion of an individual scatterer.

\section{CONCLUSION}

The results of the above calculations and estimations show that a light beam with an inhomogeneous transverse profile of intensity may enforce convection in liquids due to the transfer of momentum and torque. The phenomenon is remarkable in strongly absorbing and light-scattering materials such as colloids and liquid crystals, both presenting great interest for modern technologies and fundamental science.

\section{ACKNOWLEDGMENTS}

The authors are grateful to G. Stegeman and M. Bolshtyansky for stimulating discussions. The research was supported by U.S. Air Force Grant No. F49620-95-1-0520.
[1] P. Penfield and H. A. Haus, Electrodynamics of Moving Media (MIT Press, Cambridge, 1967).

[2] J. P. Gordon, Phys. Rev. A 8, 14 (1973).

[3] P. N. Lebedev, Rapp. près au Congres de Physique (Paris, 1900), pp. 2 and 133 [cited in R. W. Wood, Physical Optics, 3rd ed. (MacMillan, London, 1934), p. 794]; P. Lebedev, Ann. Phys. (Leipzig) 6, 433 (1901); Astrophys. J. 31, 385 (1910).

[4] E. F. Nichols and G. F. Hull, Proc. Am. Acad. 38 (1903); Phys. Rev. 13, 203 (1901) [cited in R. W. Wood, Physical Optics (Ref. [3]), p. 794].

[5] T. W. Hänsch and A. L. Schawlow, Opt. Commun. 13, 68 (1975).

[6] A. Ashkin, Science 210, 1081 (1980).

[7] M. Kerker, Am. Sci. 62, 92 (1994).
[8] E. Santamato, B. Daino, M. Romagnoli, M. Settembre, and Y. R. Shen, Phys. Rev. Lett. 57, 2423 (1986).

[9] H. He, M. E. J. Friese, N. R. Heckenberg, and R. RubinszteinDunlop, Phys. Rev. Lett. 75, 826 (1995).

[10] M. E. J. Friese, J. Enger, H. Rubinsztein-Dunlop, and N. R. Heckenberg, Phys. Rev. A 54, 1593 (1996).

[11] L. Allen, M. W. Beijersbergen, R. J. C. Speeuw, and J. P. Woerdman, Phys. Rev. A 45, 8185 (1992).

[12] S. M. Barnett and L. Allen, Opt. Commun. 110, 670 (1994).

[13] N. B. Simpson, L. Allen, and M. J. Padgett, J. Mod. Opt. 43, 2485 (1996).

[14] B. Ya. Zel'dovich, N. F. Pilipetsky, A. V. Sukhov, and N. V. Tabiryan, Pis'ma Zh. Eksp. Teor. Fiz. 31, 287 (1980) [JETP Lett. 31, 263 (1980)].

[15] I. C. Khoo and S. L. Zhuang, Appl. Phys. Lett. 37, 3 (1980). 
[16] A. S. Zolotko, V. F. Kitaeva, N. Kroo, N. N. Sobolev, and L. Csillag, Pis'ma Zh. Eksp. Teor. Fiz. 32, 170 (1980) [JETP Lett. 32, 158 (1980)].

[17] S. D. Durbin, S. M. Arakelian, and Y. R. Shen, Opt. Lett. 6, 411 (1981).

[18] I. C. Khoo and Y. R. Shen, Opt. Eng. (Bellingham) 24, 579 (1985).

[19] N. V. Tabiryan, B. Ya. Zel'dovich, and A. V. Sukhov, Mol. Cryst. Liq. Cryst. 136, 1 (1986), special issue on orientational nonlinear optics of liquid crystals.

[20] I. C. Khoo and S. Wu, Optics and Nonlinear Optics of Liquid Crystals (World Scientific, Singapore, 1993).

[21] I. Janossy et al., Mol. Cryst. Liq. Cryst. 179, 1 (1990); 203, 77 (1991); Phys. Rev. A 44, 8410 (1991); Opt. Lett. 17, 1183 (1992); Phys. Rev. E 49, 2957 (1994).
[22] I. C. Khoo, H. Li, and Yu Liang, IEEE J. Quantum Electron. 29, 1444 (1993).

[23] D. Paparo, P. Maddalena, G. Abbate, E. Santamato, and I. Janossy, Mol. Cryst. Liq. Cryst. 251, 73 (1994).

[24] S. Chandrasekhar, Hydrodynamic and Hydromagnetic Stability (Cambridge University Press, Cambridge, 1981).

[25] L. D. Landau and E. M. Lifshitz, Hydrodynamics (Pergamon, Oxford, 1989).

[26] P. G. de Gennes, The Physics of Liquid Crystals (Clarendon, Oxford, 1974).

[27] N. B. Baranova and B. Ya. Zel'dovich, Pis'ma Zh. Eksp. Teor. Fiz. 32, 636 (1980) [JETP Lett. 32, 622 (1980)].

[28] N. B. Baranova, I. V. Goosev, V. A. Krivoschekov, and B. Ya. Zel'dovich, Mol. Cryst. Liq. Cryst. 210, 155 (1992). 